\title{
Urinary Bladder Paraganglioma Unusual case with a rare Presentation A plea for Multidisciplinary Approach
}

\author{
Fahmy Abu Shawish ${ }^{1 *}$, Walid A Shawish ${ }^{2}$ \\ ${ }^{1}$ Department of Urology, Dr Suleiman al habib Hospital, Dubai, United Arab Emirates \\ ${ }^{2}$ Monash medical center, Melbourne,Victoria, Australia
}

*Corresponding Author: Fahmy Abu Shawish, Department of Urology, Dr Suleiman al habib Hospital, Dubai, United Arab Emirates.

E-Mail: Fahmi@drsulaimanalhabib.com

Received date: November 16, 2019; Accepted date: December 30, 2019; published date: January 03, 2020

Citation: Fahmy A Shawish., Walid A Shawish. (2020) Urinary bladder Paraganglioma Unusual case with a rare presentation a plea for multidisciplinary approach. Journal of Clinical Case Reports and Reviews. 1(1); DOI:10.31579/JCCR.2019/002

Copyright:@2020 Fahmy Abu Shawish, This is an open-access article distributed under the terms of the Creative Commons Attribution License, which permits unrestricted use, distribution, and reproduction in any medium, provided the original author and source are credited.

\begin{abstract}
Para ganglia is a rare bladder tumor may occur in all of the age groups. It accounts for less than $0.06 \%$ of all of the bladder tumors. It is more common in females. Typically they are extra adrenal pheochromocytoma and arise from the ganglion (Chromaffin) cells of the bladder wall. Some of them are functioning and secrets chatecholamines, others are not. Some of them are malignant and can metastasis. However approximately $10 \%$ of them possess the capacity to invade despite their lack of mitosis and cellular dissociation which usually associated with malignant tumors. We present this case because its rarity, difficult preoperative diagnosis and the lack of related proper guide lines. Currently their treatment generally is not unified. Hence it is advisable to manage such cases with a multidisciplinary approach.
\end{abstract}

Key words: paraganglioma; catecholamines; urinary bladder; metastasis,bladder; tumor resection(turbt); partial and radical cystectomy

\section{Introduction}

Chromaffin cells are neuroendocrine cells mediate the response of stress and release catecholamines regulating the circulation and respiration. Essentially they localized in the adrenal medulla and alongside the sympathetic chain [1].Tumors which arise from the adrenal medulla called pheochromcytoma. The similar extra adrenal tumor alongside the sympathetic chain called paraganglioma. The genitourinary system is rarely involved with such tumors. The urinary bladder is affected in the majority of the urinary system cases [2].Painless gross hematuria is the most common symptom. Other symptom includes persistent hypertension, headache, sweating, anxiety and micturition syncope $[3,4]$. Calcification within the tumor mimicking bladder calculi is another possible presentation [2]. A silent para ganglia is very unusual but quite dangerous as per-operative attention is not provided [5]. Commonly on CT scan it shows homogeneous or heterogeneous hyper enhancing soft tissue lesion. MRI in such cases reveal multiple areas of signal voids interspersed with hyper tense foci described as salt-pepper appearance[6].With high index of suspicion laboratory tests for catecholamines and its inactive metabolites in serum and urine as well as immunehistochemical studies particularly succinate dehydrogenase B, can help in establishing the preoperative diagnosis and hence saving the patient's life[7]. Recent advances in genetics increases our understanding of genotype-phenotype correlations which is important for both pathological diagnosis and clinical management [8]. Trans urethral bladder tumor resection may not be sufficient particularly in a lesion extended deeply into muscularis propria and even it may recur. For that reason partial or radical cystectomy with pelvic node dissection can offer a better urological outcome [9]. In metastatic (malignant) cases aggressive therapies like radical cystectomy may be essential. Nevertheless still patient may require long life follow up. However prospective studies which could assess risk and optimize management of bladder para ganglioma do not exist, thus currently pathological staging dictates the treatment [8]. Herein we present a case of nonfunctioning bladder paragangalioma and describe the clinical presentation, surgical intervention, pathological reports and clinical outcomes. We also review the literature to provide more information and understanding of this unusual disease.

\section{Case presentation}

A 56 years old male patient retired athlete, not diabetic, not alcoholic and not smoker.The main symptom makes him to seek medical advice that he lost up to $10 \mathrm{Kg}$ in the last year in spite of his regular life style. His wife noticed that he had nocturia $(2-3 \mathrm{~T} / \mathrm{N})$ and frequency of micturition which himself thought that is the normal of athlete consuming daily adequate fluids.To elicit the cause of his weight loss initially he had gastroscopy and colonscopy. Both were reported as normal. Subsequent whole body CT scan with contrast revealed a large $(5.6 \mathrm{~cm})$ well circumscribed heterogeneous and enhancing bladder base lesion (Figure 1,2).

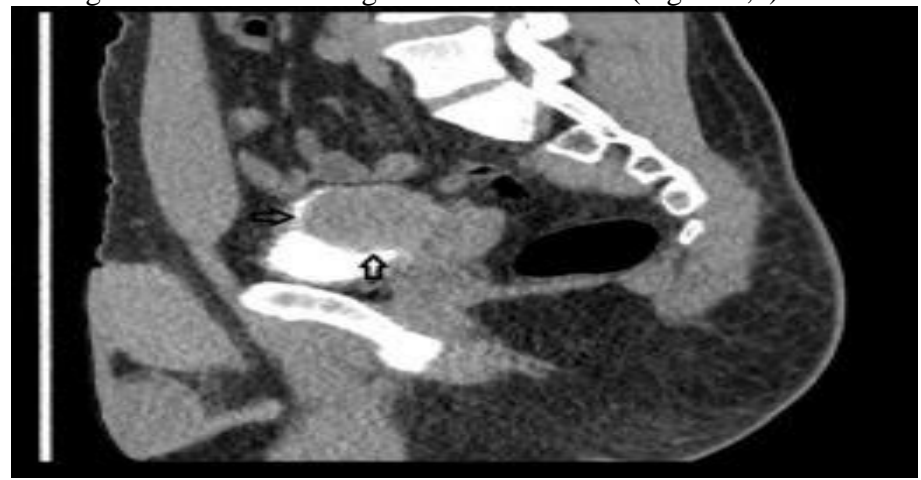

Figure 1 
J Clinical Case Reports and Reviews

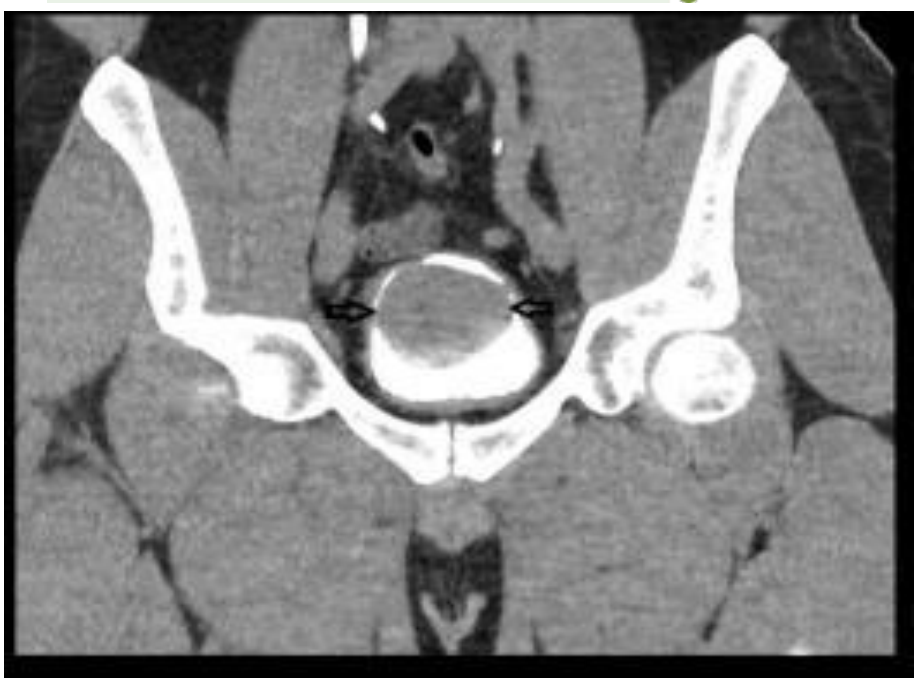

Figure 2

In addition a bilateral thyroid small nodules, $8 \mathrm{~mm}$ nodule at the lateral limb of the right adrenal gland and a small pancreatic cyst were all reported (Figure 3,4,5).

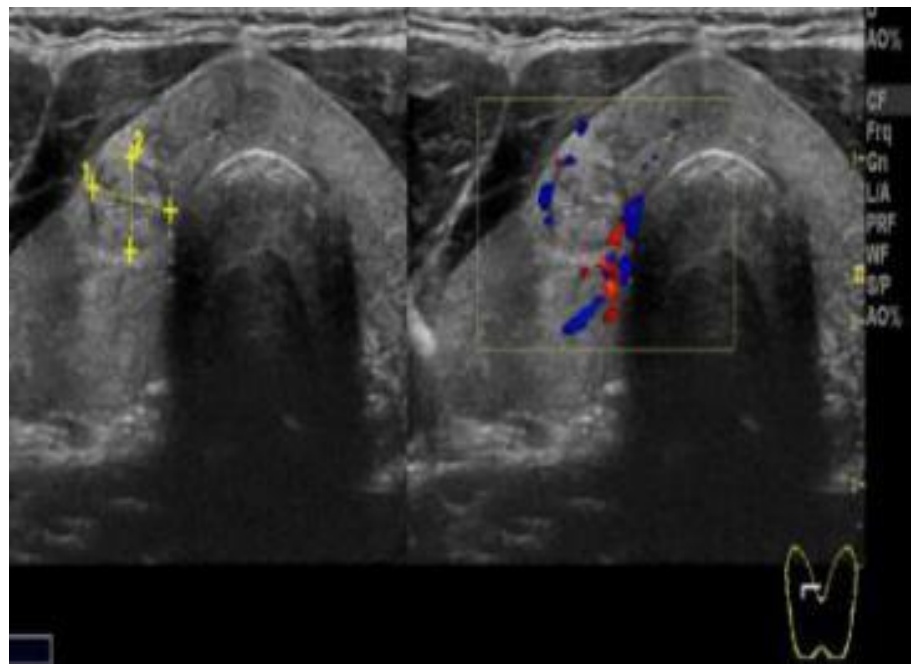

Figure 3

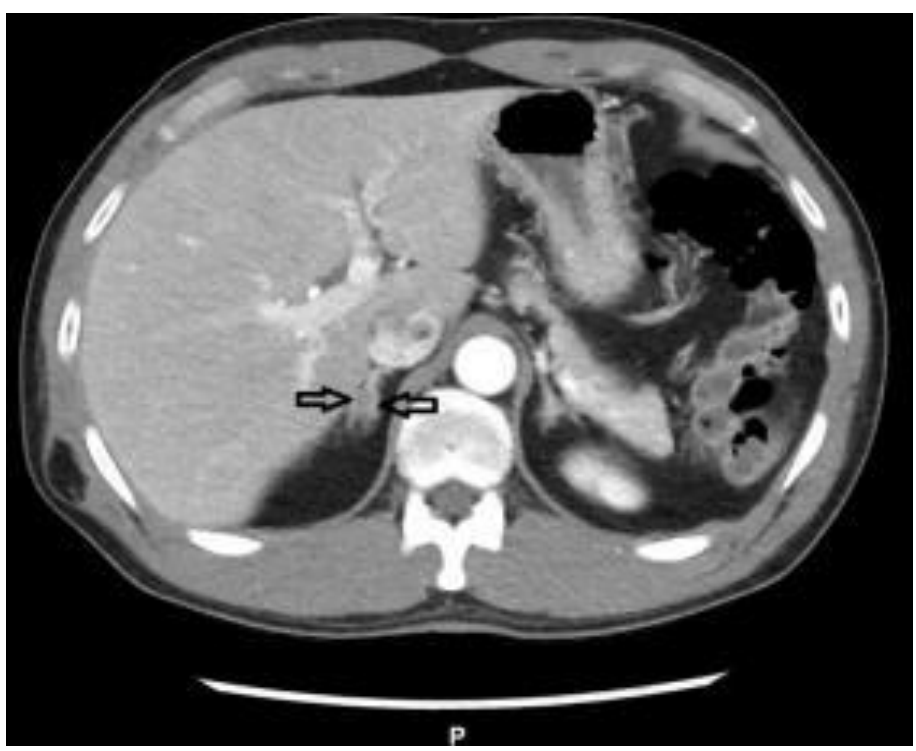

Figure 4

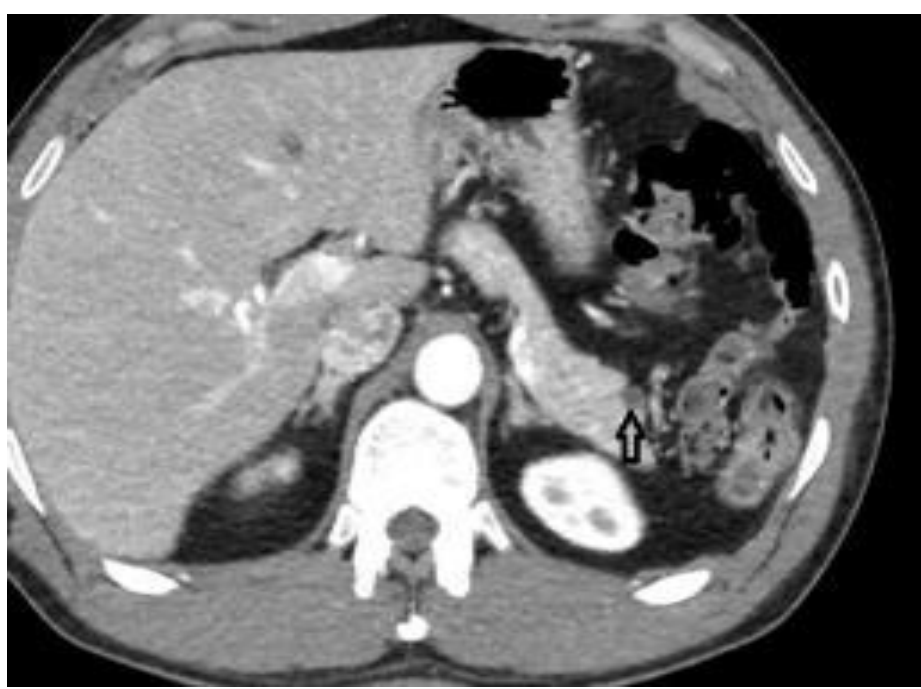

Figure 5

Urine analysis was normal (No RBCs). Blood works including CBC,kidney profile,blood sugar and liver profile were all normal.Trans urethral resection was done with almost complete clearance of the lesion(Figure 6,7).

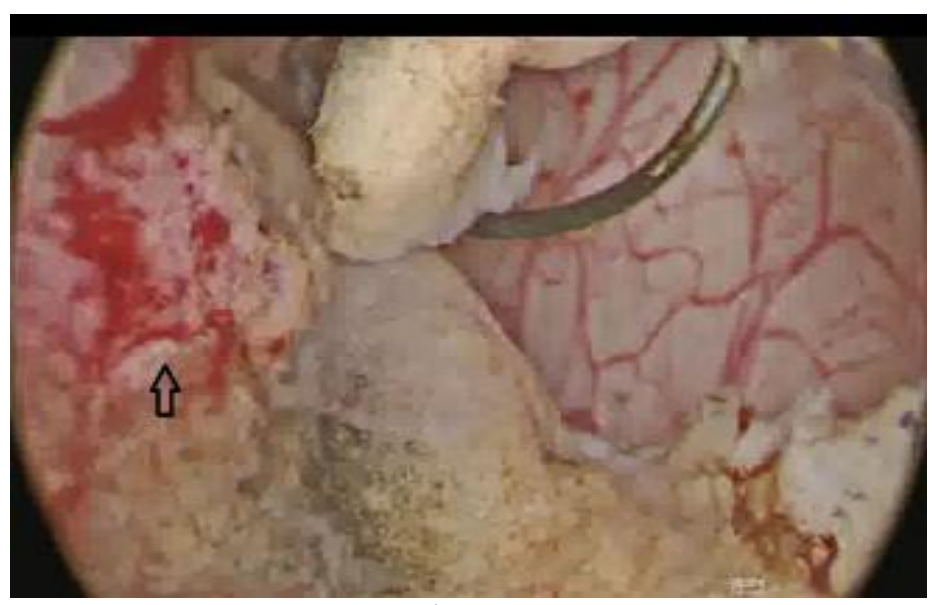

\section{Figure 6}

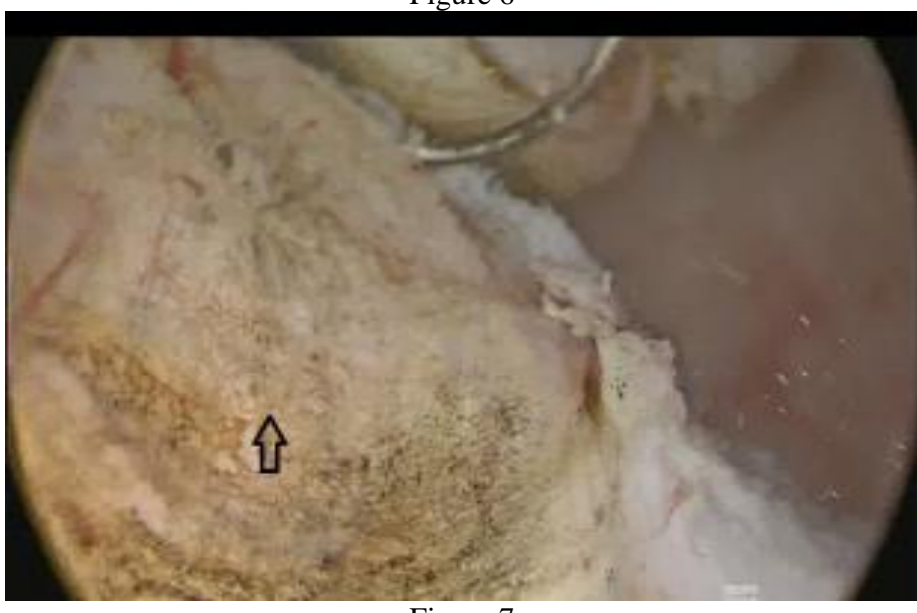

Figure 7

His blood pressure maintained on (110-130/70-90) during the surgery and even after. Post-operative follow up was uneventful. The pathologist concluded that the submitted tumor tissue which invaded the deep muscle was neither urothelial nor a prostatic carcinoma and confirmed that the morphological features although unusual are indicative of PT2 paraganglioma(Figure 8,9,10,11). 


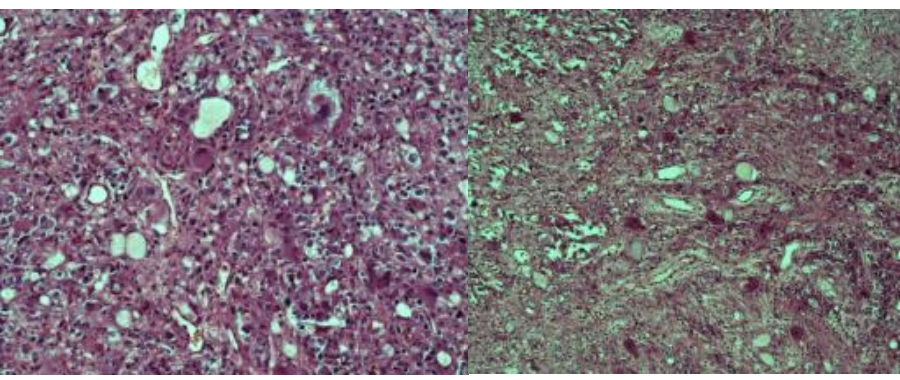

Figure 8

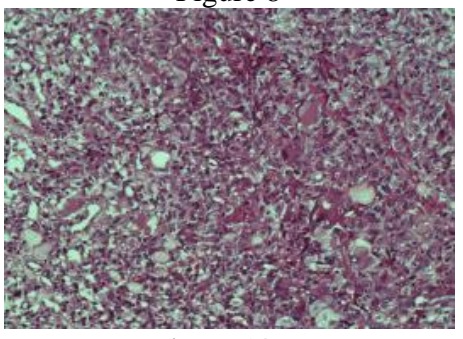

Figure 10
Figure 9

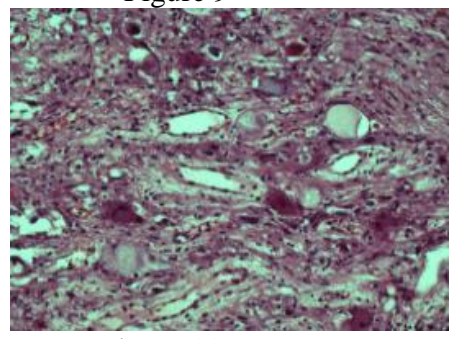

Figure 11
Two months later for taking a second opinion the patient visited a wellknown universal oncology center where his 17 years old daughter used to be followed for pancreatic cancer treatment. Eventually he had another cystoscopy with a biopsy taken from the same site of the previously resected bladder lesion.The diagnosis of paraganglionoma pathologically was confirmed separately with this second biopsy. However the patient was recommended to have three monthly cystoscopy. A suggestion which he was not very welling to proceed with. In the other hand thyroid nodule aspirated with fine needle and the result revealed benign follicular nodule consistent with cystic degeneration (Figure 3).Subsequent yearly urinary bladder ultrasound for two successive years were reported as normal (Figure 12, 13).

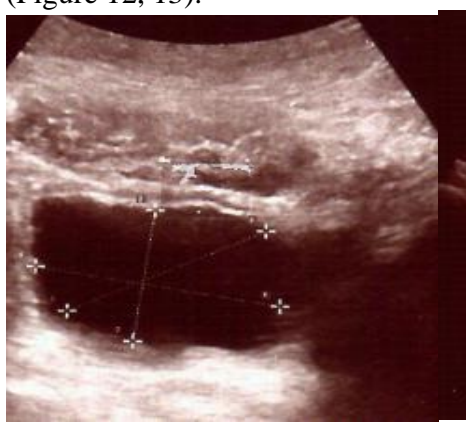

Figure 12

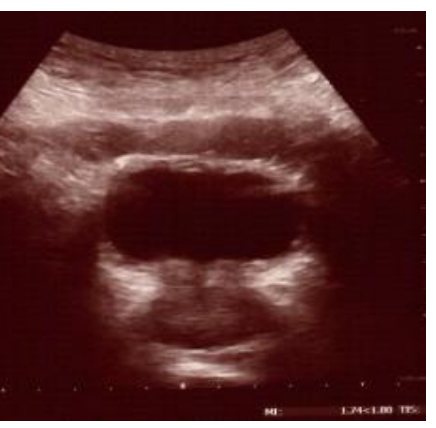

Figure 13
Three years after the initial resection, real time ultrasound scan and contrast CT scan abdomen and pelvis revealed $1 \mathrm{~cm}$ intramural lesion encircling the lower portion of the right ureter, otherwise bladder was normal(Figure 14,15).

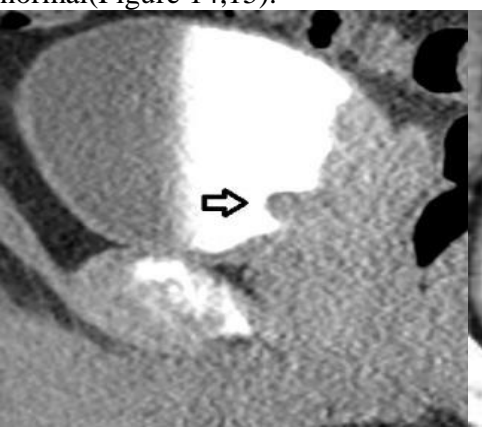

Figure 14

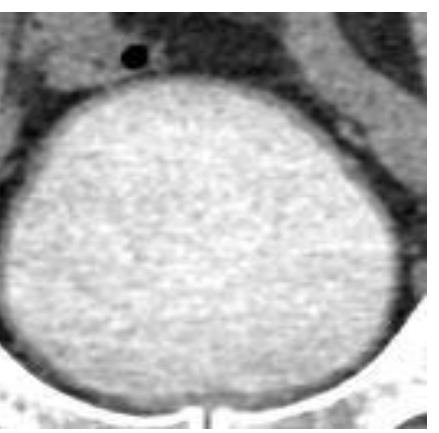

Figure 15
The right ureter and the right kidney both were normal. No regional recurrences and no local or distant lymph nodes metastasis were seen. Meanwhile Normetanephrine get raised (416ug/24hrs) and simultaneously Metanephrines, fraction, plasma free also get raised (99ug). Depending on these changes partial cystectomy with right ureter re implantation was strongly recommended. The patient was not very eager to proceed, at least, at this stage while he is still clinically free of any related symptom.

Dr Priti Joshi MD concluded: This tumor is neither a urothelial nor a prostatic carcinoma. The morphological features, although Unusual are indicative of a paraganglioma (a type of neuroendocrine tumor). This is further supported by

Immunohistochemistry as the tumor cells shows diffuse and strong positive staining with neuroendocrine markers

-synaptophysin and chromogranin and S100 positive sustentacular cells are seen in the better preserved areas where the

tumour morphology is nested. The tumor stains negative with epithelial markers - AE1/AE3, CAM 5.2, CK 7, CK 20,

$34 B E 12$ and p63, thus excluding a carcinoma; negative for melanoma markers - HMB-45 and MelanA and negative for

Prostate carcinoma markers-PSA and PSAP. The large cells atypical cells described as having abundant eosinophilic

Cytoplasm stain positive with S100 in some areas and negative in others. In my opinion, these cells represent, both

Degenerative atypia and the S100 positive large cells are ganglion cells. As ganglion cells are also a component of the

tumour, the most appropriate designation for this entity is a composite paraganglioma.

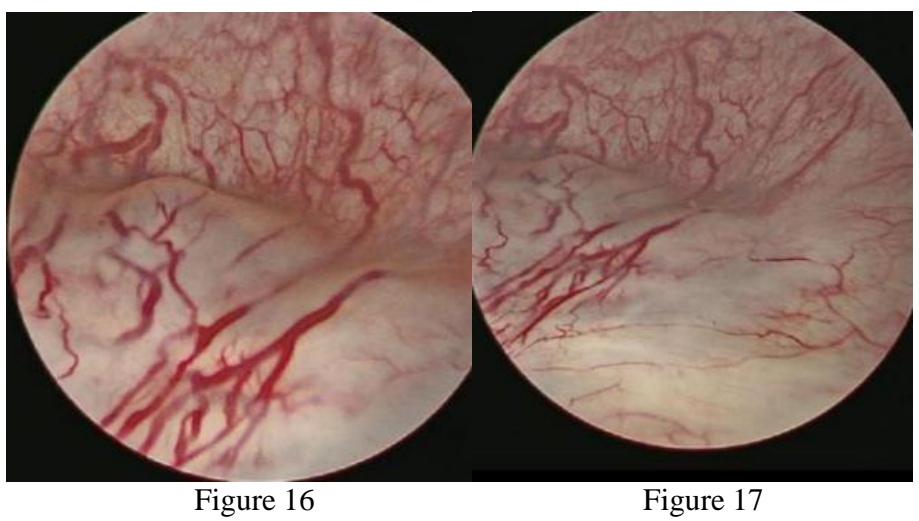

\section{Discussion}

Bladder paraganglioma is a rare tumor, often originates in the bladder trigone and rarely in the anterior or lateral wall, which was exactly the same as in our presented case [9]. It should be taken in consideration that up to $50 \%$ of paraganglia are hereditary and can be associated with type 1 neurofibromatosis and familial paraganglia[10]. It can be metastatic, a nomination given recently by WHO to this type of the tumor previously was known as malignant paraganglioma[8,11]. However only when the primary tumor infiltrates locally into the non-nervous system or distant organs without embryo residual ganglia such as liver,lungs,spleen,brain ,bones and lymph nodes can the diagnosis of malignancy be made[12].Despite its potential lethality local invasion alone is a poor predictor of metastasis[8].The para ganglia tumor often metastasize late and their behavior is unpredictable.They have high recurrence rate regardless of the given treatment modal[4]. Nevertheless such tumor never be signed out as histologically benign. Equally they should not be signed out as malignant unless unequivocal documentation of distant metastasis with or without lymph node were confirmed [8]. Functional 
paraganglia are frequently malignant [13]. Transurethral resection of bladder tumor (TUR BT) might be an option in cases in which the tumor has not invaded the surrounding fat or in a periureteral orifice lesions $[9,14]$. In our presented case we have done TURBT on a mass unexpectedly detected preoperatively in a computer tomography (CT) with contrast scan. Subsequently the tumor was diagnosed pathologically as a nonfunctional paraganglioma. In our search in the English literature we found partial and even radical cystectomy frequently has been recommended. Radiotherapy attempted under special condition [15]. In one occasion the tumor initial resection was accomplished laparascopically[6,16]. Regardless the given treatment patient may require long term follow up, three monthly as suggested by V.Priyadarshi or annually as suggested by C.C.Tsai $[5,17]$. Obviously at present there is no definite guide lines to be considered in such cases.

\section{Conclusion}

Paraganglioma is a peculiar and rare endocrine bladder tumor presented with a different phenotype. Despite the accumulated pathological, radio logical and clinical knowledge about the tumor, its management is not optimized and the guidelines are not accomplished. At present mostly each case managed by its own merits. However when such cases are encountered we expect multidisciplinary approach may facilitate the treatment.

\section{Acknowledgment}

Said Al Shakh MD, Ahmad Albani MD,Ghaida Kaddaha MD, Wessam Bou-Assaly MD, Priti Joshi MD and Simon K Sfeir MD

For their input in diagnosis and management of the case

\section{References}

1. Kouba E, Cheng L. (2016) Neuroendocrine tumor of the urinary bladder according to the 2016 World health organization classification Molecular and clinical characteristics Endocr Pathol; 27(3) PP:188-199.

2. Sk Barua, N Gupta, TP Rajeev, SJ Bara. (2015) Atypical presentation of vesicle paraganglioma Urology Nephrology open access Journal; 2(5), 00053

3. Georgios Tsanboukas, Vasilios Vossos, Kristiana Gkeka, Loanna Kotsi Kogianni loanna.(2018) Paraganglion of urinary bladder
Hellenic Urology: 29(4),

4. Deng JH, Li HZ, Zhang Ys, Liu GH. (2010) Paragangliomas of the urinary bladder: a report of 9 cases Chin J cancer Aug; 29(8) 729734

5. V Priyadarshi, Dilip Kumar Pal. (2015) Paraganglionoma of the urinary bladder Uro Ann, ,7(3): 402-404

6. Gundeti Dwethi, Chnnamaneni and Tippani Ramesh.(2019)Urinary bladder paraganglioma,48(3):48(3)-48(c)

7. Genta I Wamoto Uemura Parananglion in the bladder, a case report J of Medical Case Reports 2017;11: 306

8. Arthur S., Tischler Archives of pathology and laboratory medicine Vol 132 issues 8, August 2008

9. Yi=Kai Chang, I-Ni Chiang, Chung-Hsin Chen, Shuo-Meng Wang, Yuan-Ju Lee, Yeong-Shiau Pu, Chao-Yuan Huang Paraganglon of the urinary bladder: A report of 6 cases Urological Science Volume 26, issue 2, June 2015 pp 111-114

10. Young WFJ Paraganglomas: Clinical overview Annals of the New York Academy of sciences 2006;1073(1): 21-29

11. Lam AK Update on Adrenal Tumors in 2017 World Health organization (WHO) of Endocrine Tumors Endocr Pathol, 2017 Sep; 28(3):213-227

12. M. Zhou, J.I. Epistein, R.H. Young Paraganglioma of the urinary bladder: A lesion that may be misdiagnosed as urothelial carcinoma in transurethral resection specimen AJSP,28(2004),PP:99-100

13. O Riordian DS, Young WF Jr. Grant CS, Carney JA, Van Heerden JA(1996) Clinical spectrum and outcome of functional extra adrenal paraganglioma World J surgery Sep; 20(7): 916-922

14. Genta Iwamoto, Takashi Kawahara, Mikiko Tanabe, Sahoko Ninomiya, Daiji Takamoto, Taku Mochi(2017) Zuki Paraganglioma in the bladder: A case report $\mathbf{J}$ of medical case reports 11, Article Number: 306.

15. Dong You, Ruizhen Ren, Ercheng Cheu, Shulin Chen, Dawei Wang, Jianhui Liu.(2016) Radiotherapy for urinary bladder pheochromocytoma With invasion to the prostate: A case report and literature review Mol Clin Oncol June; 4(6). pp1060-1062

16. Divya Ratna Dhawan, Arivind Ganpule, Veermani Muthu, Mahesh $\mathrm{R}$ Desai. (2008) Laparascopic Managment of Calcified paraganglioma of bladder Urol J; 5:126-8

17. C.C. Tsai, W.J. Wu, K.S. Chueh, W.M. Li, C.H. Huang, C. (2011)C. Wu, et al Paraganglioma of the urinary bladder first presented by bladder bloody tamponade: two cas reports and review of literatures Kaohsiung J Med Sci,27, PP. 108-113 